\section{Efficacy of adalimumab in moderate to severe hidradenitis suppurativa: Real life data}

\author{
Aikaterini Kyriakou, ${ }^{1}$ \\ Anastasia Trigoni, ${ }^{1}$ Nikiforos Galanis, ${ }^{2}$ \\ Dimitrios Sotiriadis, ${ }^{1}$ \\ Aikaterini Patsatsi ${ }^{1}$ \\ ${ }^{1}$ Second Department of Dermatology \\ and Venereology, Aristotle University of \\ Thessaloniki, General Hospital \\ "Papageorgiou"; ${ }^{2}$ Division of Sports \\ Medicine, Department of Orthopedics, \\ Medical School, Aristotle University of \\ Thessaloniki, Greece
}

\begin{abstract}
Hidradenitis suppurativa (HS) is a relapsing, inflammatory disease characterized by painful nodules, abscesses, sinuses track formation and scarring. HS has a great impact on patients' quality of life and its treatment may be really challenging. Adalimumab provides a new therapeutic option for HS. Our aim was to assess the therapeutic potential of adalimumab on patients with HS based on the data from the daily clinical practice of an HS Outpatient Clinic. 19 patients with clinically evident moderate to severe HS, under adalimumab treatment for at least 24 week, participated in this observational, retrospective study. The Hidradenitis Suppurativa Physician's Global Assessment scale, Modified Santorius scale and Dermatology Life Quality Index (DLQI) at baseline, week 4, week 12 and week 24 were retrieved from the records. Both Modified Santorius score and DLQI were significantly decreased during the weeks of evaluation (Friedman's test; $\mathrm{P}<0.001)$. The proportion of patients who achieved clinical response was $10.5 \%$ $(\mathrm{n}=2)$ at week 4, 42.1\% $(\mathrm{n}=8)$ at week 12 and $63.2 \%(\mathrm{n}=12)$ at week 24 . Treatment with adalimumab was linked with both clinical remission of HS and improvement of patients' quality of life.
\end{abstract}

\section{Introduction}

Hidradenitis suppurativa (HS), which is also referred to as acne inversa, is a chronic, relapsing, inflammatory disease. ${ }^{1}$ It presents with non-inflamed or inflamed nodules, abscesses, sinuses track formation and scarring development mainly at the anguine and axilla. ${ }^{2}$ It most commonly effects women and it is highly associated with smoking and overweight. ${ }^{3}$

HS has a great impact on patients' quality of life. ${ }^{4}$ Apart from pain, malodor and discomfort, which can definitely cause, HS can lead to depressive symptoms, feelings of hopelessness and low self-esteem. ${ }^{5}$ Mild disease can be usually managed with topical agents; however, progression to moderate or severe disease requires the use of systemic therapies such as oral antibiotics, retinoids and immunosuppressant drugs. ${ }^{6}$ Even though there are therapeutic options, management of HS is challenging, since there are plenty of cases not responsive to treatment. ${ }^{7}$ Therefore, the approval of adalimumab for the treatment of moderate to severe HS enriched the therapeutic armamentarium and was proven to be promising for refractory HS cases. ${ }^{8}$

Despite the well-designed studies, real life data expressing the experience of adalimumab in HS cases are limited..$^{9-13}$ Therefore, this study was designed to assess the therapeutic potential of adalimumab on patients with HS based on the data from the daily clinical practice of an HS Outpatient Clinic, located in a Tertiary Hospital in Northern Greece.

\section{Materials and Methods}

A single-center, observational, retrospective study was conducted to assess the efficacy of adalimumab on patients suffering from HS in daily practice. The records of HS patients who visited our department between February 2016 and May 2017 were used. Eligibility criteria were clinically evident moderate to severe HS, with the Hidradenitis Suppurativa Physician's Global Assessment (HS-PGA) scale $\geq$ moderate, under adalimumab treatment for at least 24 weeks. Subject who had to discontinue treatment with adalimumab due to adverse event or had to receive an adjuvant therapeutic modality (except for topical agents) were excluded. Written informed consent from all patients was provided. The study design scheme is presented in Figure 1. All patients were treated with subcutaneous injections of adalimumab at a dose of $160 \mathrm{mg}$ at baseline, $80 \mathrm{mg}$ at week 2, $40 \mathrm{mg}$ at week 4 and $40 \mathrm{mg}$ weekly thereafter. Patients' age, gender, age at disease onset, time to final diagnosis, Body Mass Index (BMI), waist circumstance, occupational and educational status, presence of arthritis, dyslipidemia, diabetes mellitus and hypertension, systolic and diastolic blood pressure, work absenteeism (yes/no), days of absence per year, treatment history and
Correspondence: Aikaterini Kyriakou, Second Department of Dermatology and Venereology, General Hospital "Papageorgiou”, Nea Eflkarpia, Ring Road, 564 03, Thessaloniki, Greece.

Tel.: +30.6932.719606 - Fax: +30.2310.991473. E-mail: docmouli@gmail.com

Key words: Adalimumab; Hidradenitis suppurativa; Acne inversa.

Contributions: the authors contributed equally.

Conflict of interest: the authors declare no potential conflict of interest.

Funding: none.

Received for publication: 31 August 2018. Accepted for publication: 19 September 2018.

This work is licensed under a Creative Commons Attribution-NonCommercial 4.0 International License (CC BY-NC 4.0).

(C) Copyright A. Kyriakou et al., 2018

Licensee PAGEPress, Italy

Dermatology Reports 2018; 10:7859

doi:10.4081/dr.2018.7859

Hurley stage were recorded at baseline. The HS-PGA scale, Modified Santorius scale and Dermatology Life Quality Index (DLQI) at baseline, week 4, week 12 and week 24 were retrieved from the records. Roughly, HS-PGA scale assigns patients to one of six ordinal categories (clear, minimal, mild, moderate, severe, or very severe) based on the number of nodules (inflammatory and non-inflammatory), abscesses, and fistulas (draining and non-draining); ${ }^{8}$ the Modified Sartorius Scale assesses the number of involved anatomical regions, the number and type of lesions and the extent of involvement, ${ }^{14}$ while DLQI measures dermatology specific health-related quality of life. The presence of clinical response, which was defined as an HS-PGA score of clear, minimal, or mild with at least a 2grade improvement relative to baseline score, was assessed at week 4, week 12 and week 24.

The primary efficacy endpoints were i) the percentage of patients who achieved clinical response at week 4, week 12 and week 24 and ii) the proportion of patients at each stage of the HS-PGA scale at baseline, week 4, week 12 and week 24. Major secondary endpoints included i) the assessment of the Modified Santorius scale at baseline, week 4, week 12 and week 24 , as well as the detection of possible, statistically significant differences in the Santorius scale through the evaluation period and ii) the assessment of DLQI at baseline, week 4, 
week 12 and week 24 and the detection of possible, statistically significant differences in DLQI through the evaluation period.

\section{Statistical analysis}

The software Statistical Package for Social Sciences (SPSS), version 22.0 (SPSS, Inc., Chicago, IL, USA) was used for the statistical analysis of the data. Shapiro-Wilk test was performed to test the normality of continuous variables. Descriptive statistics were used to describe the study population's demographic and clinical characteristics. All continuous variables were expressed as the mean \pm standard deviation, median and range. The categorical variables were presented as frequencies and percentages. Friedman's ANOVA test was used to detect differences in the Modified Santorius scale and DLQI across the whole follow-up period, as well as the Bonferroni post hoc test. All tests were two sided and the significance level was $\mathrm{a}=$ 0.05 .

\section{Results}

In total, 19 patients participated in the study. Patients' demographic and clinical characteristics at baseline are summarized in Table 1.

Both Modified Santorius score and DLQI were significantly decreased during the weeks of evaluation (Friedman's test; P $<0.001)$. All data are presented in Table 2 and Figure 2. The proportion of patients who achieved clinical response was $10.5 \%$ $(\mathrm{n}=2)$ at week $4,42.1 \%(\mathrm{n}=8)$ at week 12 and $63.2 \%(\mathrm{n}=12)$ at week 24 (Figure 3). Data regarding the proportion of patients at each stage of the HS-PGA from baseline to week 24 are presented in Figure 4. At baseline, all patients suffered from moderate or severe disease, as this was assessed with the HS-PGA scale. At week 24, only $5.3 \%$ of the patients had moderate disease; the rest (94.7\%) achieved an HS-PGA score of mild, minimal or clear.

\section{Discussion and Conclusions}

This study provided evidence that adalimumab is an efficient and safe treatment option for moderate to severe HS. Both Modified Santorius score and DLQI were significantly decreased during the weeks of evaluation, while $63.2 \%$ of patients achieved clinical response by week 24 . HS is a chronic, painful, inflammatory, skin disease which is frequently refractory to treatment. Its pathogenesis is not completely understood but immunologic abnormalities
Table 1. Patients' demographic and clinical characteristics at baseline.

\begin{tabular}{lc} 
Characteristics & Statistics \\
Age (years) & \\
Mean \pm SD & $39.05 \pm 11.65$ \\
Median (Min-Max) & $40.0(21.0-59.0)$ \\
Gender & $14(73.7)$ \\
Male n (\%) & $5(26.3)$ \\
Female n (\%) & $28.11 \pm 8.13$ \\
\hline Age at disease onset (years) & $27.0(15.0-40.0)$ \\
Mean \pm SD & \\
Median (Min-Max) & $63.82 \pm 78.57$ \\
Time to diagnosis (months) & $24.0(0.1-264.0)$ \\
Mean $\pm S D$ & \\
Median (Min-Max)
\end{tabular}

\section{BMI $\left(\mathrm{kg} / \mathrm{m}^{2}\right)$}

Mean \pm SD

$32.74 \pm 9.92$

Median (Min-Max)

$29.41(22.96-60.96)$

\begin{tabular}{lc} 
Waist circumstance $(\mathrm{cm})$ & $103.89 \pm 22.25$ \\
Mean \pm SD & $98.0(67.0-150.0)$ \\
Median (Min-Max) & \\
\hline
\end{tabular}

Occupational status

Full time n (\%) $13(68.5)$

On sick leave $n(\%) \quad 2(10.5)$

On disability benefit n (\%) $2(10.5)$

Retired n (\%) $2(10.5)$

Educational status

$\begin{array}{ll}\text { High school n (\%) } & 13(68.4) \\ \text { University }(4 \text { years) n (\%) } & 5(26.3)\end{array}$

$\begin{array}{lr}\text { University (4 years) n (\%) } & 5(26.3) \\ \text { University (> } 4 \text { years) } \mathrm{n}(\%) & 1(5.3)\end{array}$

Arthritis

Yes n (\%) $1(5.3)$

$\begin{array}{lr}\text { Non }(\%) & 18(94.7)\end{array}$

Dyslipidemia
Yes n (\%)

Non (\%) $17(89.5)$

Diabetes mellitus

Yes $\mathrm{n}(\%) \quad 3(15.8)$

Non (\%) $16(84.2)$

Hypertension

Yes $\mathrm{n}(\%) \quad 10(52.6)$

$\begin{array}{ll}\text { Non } n(\%) & 9(47.4)\end{array}$

Systolic BP (mmHg)

Mean \pm SD $\quad 130.26 \pm 15.23$

$\begin{array}{ll}\text { Median (Min-Max) } & 130.0(110.0-170.0)\end{array}$

Diastolic BP $(\mathrm{mmHg})$
$\quad$ Mean \pm SD

Median (Min-Max) $\quad 75.0(65.0-90.0)$

Smoking status

Yesn (\%) $14(73.7)$

Non $(\%) \quad 5(26.3)$

Work absenteeism
Yes $\mathrm{n}(\%)$

Non $(\%) \quad 8(42.1)$

Days of absence per year

Mean \pm SD $\quad 4.68 \pm 6.92$

Median (Min-Max)

$4.0(0.0-30.0)$

Previous treatments

Topical and systemic antibiotics n (\%) 14 (73.7)

Systemic retinoid n (\%) $2(10.5)$

Systemic antibiotics + retinoid n (\%) $3(15.8)$

Hurley stage

Stage II n (\%) $14(73.7)$

Stage III n (\%) 5 (26.3) 
have been suggested to play a causal role in the disease. ${ }^{15}$ IL-1 $\beta$, TNF- $\alpha$ and IL-10 levels are elevated in HS skin providing data that biologics targeting these cytokines may be beneficial in HS. ${ }^{16}$

As treatment of HS can be really challenging, various therapeutic options have been suggested with multifarious results. Topical or systemic antibiotics, systemic steroids, antiandrogens, retinoids, biological drugs and surgical or laser excision of the affected areas are mainly the options in regard to the severity of the disease. ${ }^{17}$

Biological agents (adalimumab, infliximab, ustekinumab) could be a therapeutic option for patients suffering from moderate to severe HS. ${ }^{13,18}$ Partial responses are achieved in about $50 \%$ of patients, while the lack of response to one particular biologic agent does not preclude a potential efficacy to another one. ${ }^{18}$ Several biologic agents have been used for the treatment of severe or recalcitrant to treatment HS. ${ }^{19,20}$ However, adalimumab, which is a fully human, IgG1 monoclonal antibody specific for TNF- $\alpha$, is the only biologic agent that has been approved for the treatment of moderate to severe HS. ${ }^{21}$

In a prospective, open study 15 patients suffering from moderate-to-severe HS, were treated with adalimumab at the indicated dose. Sartorius score was significantly reduced by week 24 , as well as the VAS score and DLQI. ${ }^{22}$ Moreover, some case series have been published on the use of adalimumab (at the standard regime for psoriasis vulgaris) for the treatment of HS; all studies reported significant improvement in the number of affected regions, nodules, fistulas and DLQI after 4-6 weeks of treatment. ${ }^{23-25}$ Sbidian et al. conducted a national cohort study and reported that $71.4 \%$ of the patients receiving adalimumab achieved a partial response and none had a complete response after a median follow-up of 6.8 months. ${ }^{13}$ However, another prospective, open study with 6 patients with moderate to severe HS, treated with adalimumab failed to show statistically significant improvement of the both HS Severity Index (HSSI) and DLQI by week $12 .{ }^{9}$

In a prospective, randomized, double- blind, placebo-controlled study, 21 patients with HS received adalimumab $(80 \mathrm{mg}$ s.c. at baseline, followed by $40 \mathrm{mg}$ s.c. every other week for 12 weeks). A significant improvement in Santorius score was achieved at 2 weeks $(\mathrm{P}<0.024)$ but not at the end of treatment $(\mathrm{P}=0.07) .{ }^{26}$ In a Phase 2, randomized, double-blind, placebo-controlled study, $17.6 \%$ of patients treated with $40 \mathrm{mg}$ adalimumab weekly, achieved clinical response at week 16 and the drug was well-tolerated. ${ }^{8}$ These results were confirmed by two phase 3, multicenter, double-blind, placebo-controlled trials of adalimumab for HS, PIONEER I and PIONEER I. ${ }^{21}$ Clinical response rates were significantly higher in the adalimumab groups as compared to placebo at week $12 ; 41.8 \%$ versus $26.0 \%$ (P $=0.003)$ in PIONEER I and $58.9 \%$ versus $27.6 \%(\mathrm{P}<0.001)$ in PIONEER II. ${ }^{21}$ The

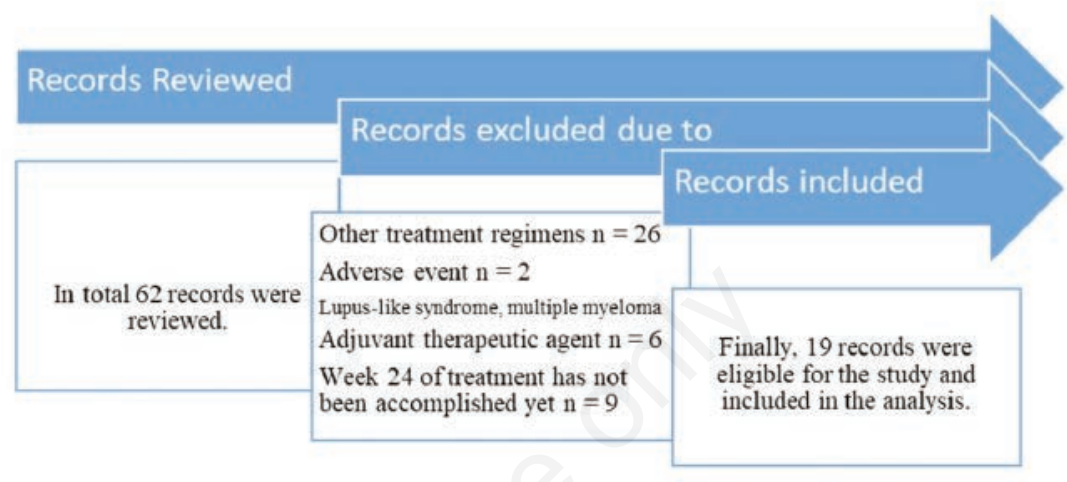

Figure 1. Study design scheme.

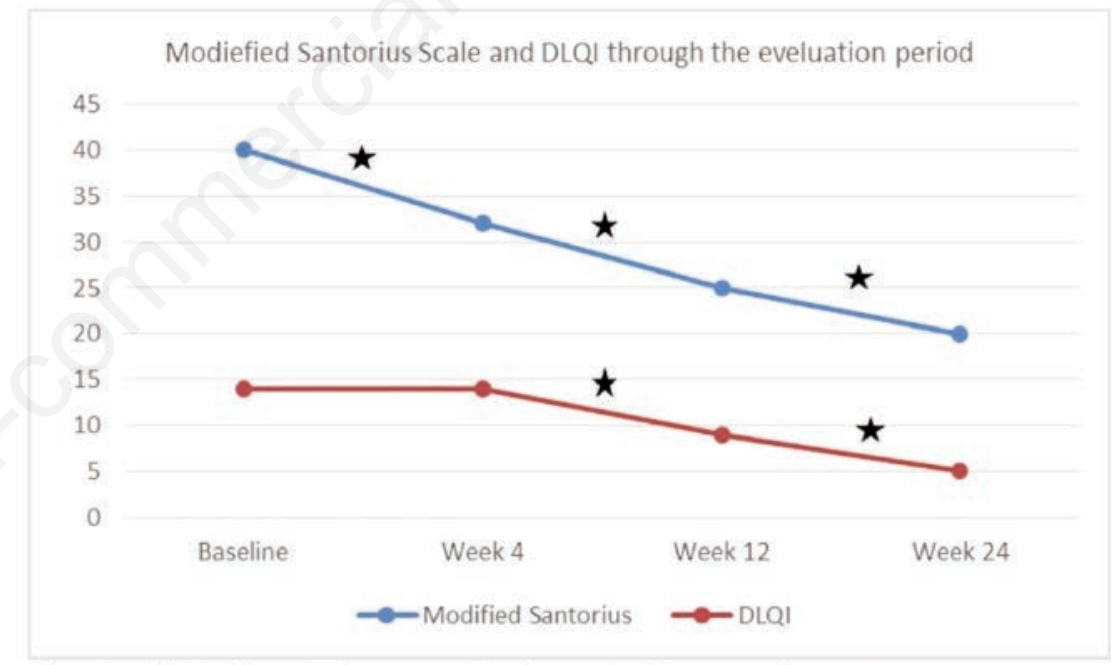

*Statistically significant, Wilcoxon signed-rank test; Bonferroni post hoc test

Figure 2. Modified Santorius Scale and Dermatology Life Quality Index (DLQI) through the evaluation period.

Table 2. Descriptive statistics for modified Santorius scale and Dermatology Life Quality Index (DLQI) during weeks of evaluation.

\begin{tabular}{lcccc} 
Variables & Baseline & Week 4 & Week 12 & Week 24 \\
Modified Santorius Scale & & & \\
$\quad$ Mean \pm SD & $42.21 \pm 18.42$ & $35.26 \pm 16.14$ & $26.26 \pm 12.01$ & $19.37 \pm 7.95$ \\
Median (Min-Max) & $40.0(15.0-80.0)$ & $32.0(14.0-80.0)$ & $25.0(10.0-60.0)$ & $20.0(6.0-34.0)$ \\
DLQI (0-30) & & & $10.47 \pm 4.89$ & $6.05 \pm 5.23$ \\
Mean \pm SD & $17.00 \pm 6.36$ & $15.63 \pm 5.95$ & $9.0(0.0-20.0)$ & $5.0(0.0-20.0)$ \\
Median (Min-Max) & $14.0(5.0-25.0)$ & $14.0(9.0-26.0)$ & & $<0.001^{*}$ \\
\hline
\end{tabular}


rates of serious adverse events did not differ significantly in the study groups, suggesting the good safety profile of the drug. ${ }^{21}$ Moreover, the safety of the drug in treating HS is verified by a systematic review which has shown no significant difference in serious or infectious adverse effects compared with placebo. ${ }^{27}$

It seems that reduction of adalimumab treatment frequency from every week to every other week, may lead to deterioration of HS in some patients. ${ }^{8}$ Moreover, serious adverse effects, which are not common, seem to have a similar pattern in both dosage groups. In patients with HS, the safety of adalimumab weekly and every other week has been proven to be comparable. ${ }^{11}$ The safety of the weekly administered adalimumab in patients with dermatologic conditions is supported by data on other non-dermatologic conditions, such as rheumatoid arthritis or Crohn's disease. ${ }^{11}$ Therefore, adalimumab treatment for HS is recommended on a weekly basis. .7,19,21 $^{1}$

Since HS has a great psychological impact and is associated with pain, depression and anxiety, ${ }^{28}$ evaluation of therapeutic interventions for HS should not only focus on objective assessments, but also on subjective patient-reported outcomes. ${ }^{29}$ It has been shown that adalimumab 40mg s.c. weekly, apart for the HS lesions, improves both pain and depressive symptoms. ${ }^{8,29} \mathrm{~A}$ recent systematic review suggests that adalimumab $40 \mathrm{mg}$ weekly improves quality of life compared with placebo, with a reduction in DLQI score of 4.0 points. ${ }^{27}$ Concerning the dynamic evaluation of a treatment response, the most commonly used measures are the Modified Sartorius

\section{Clinical response rates to adalimumab through the evaluation period}

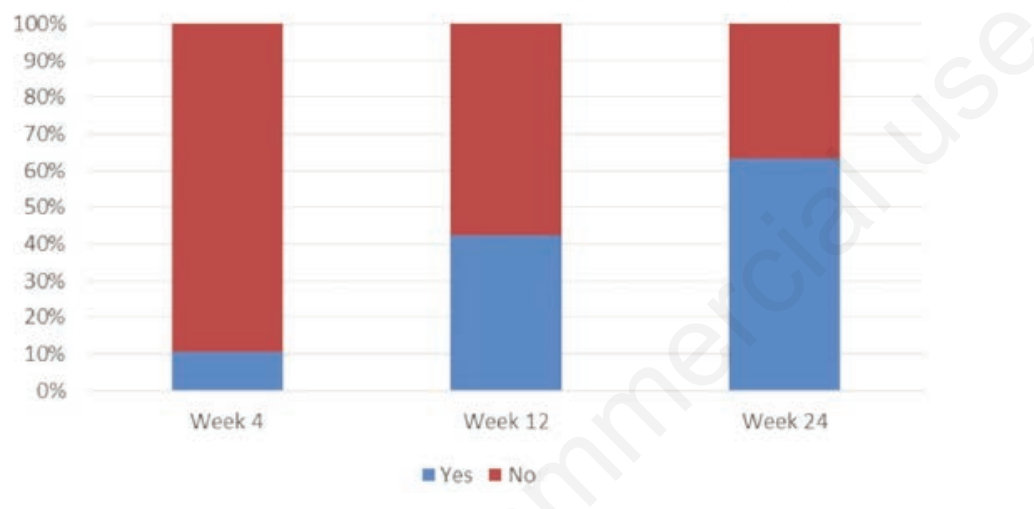

Figure 3. Clinical response rates to adalimumab through the evaluation period.
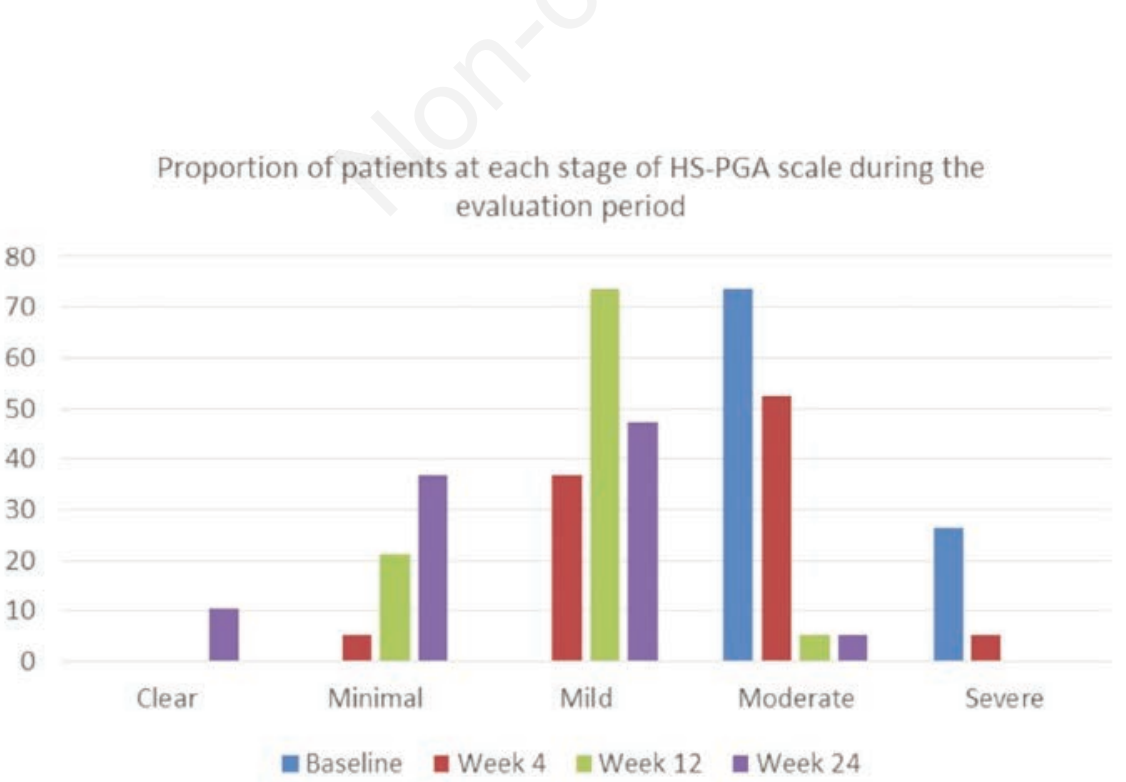

Figure 4. Proportion of patients at each stage of the Hidradenitis Suppurativa Physician's Global Assessment (HS-PGA) scale during the evaluation period.

Score $^{14}$ and the HS-PGA. ${ }^{8}$ Recently, Hidradenitis Suppurativa Clinical Response (HiSCR) has been developed to simplify the evaluation process and increase the sensitivity to detect HS-specific lesions. ${ }^{30}$ HiSCR has been shown to be more responsive in detecting changes in response to treatment than HS-PGA. ${ }^{31}$ However, the use of HiSCR as the primary end point in clinical trials has been questioned, since this score only measures the inflammatory component of HS (nodules and abscesses) which is obviously reduced by anti-inflammatory medication. ${ }^{10}$

This study showed the high efficacy of adalimumab in a series of Greek patients with moderate to severe HS, through a 24week period. Treatment with adalimumab was linked with both clinical remission of HS, as this was measured by Modified Santorius score and HS-PGA scale, and improvement of patients' quality of life. Moreover, the good safety profile of the drug, which is well-displayed in literature, was verified. To achieve healing in HS, adalimumab may need to be combined with other treatments such as antibiotics and surgical excision of persisting lesions. ${ }^{17,19}$ Either as monotherapy or adjuvant therapy, adalimumab enriches the therapeutic armamentarium for HS, the treatment of which may be really challenging.

\section{References}

1. Alikhan A, Lynch PJ, Eisen DB. Hidradenitis suppurativa: a comprehensive review. J Am Acad Dermatol 2009;60:539-61.

2. Scheinfeld N. An atlas of the morphological manifestations of hidradenitis suppurativa. Dermatol Online J 2014;20:22373.

3. Revuz JE, Canoui-Poitrine F, Wolkenstein P, et al. Prevalence and factors associated with hidradenitis suppurativa: results from two case-control studies. J Am Acad Dermatol 2008;59: 596-601.

4. Esmann S, Jemec GB. Psychosocial impact of hidradenitis suppurativa: a qualitative study. Acta Derm Venereol 2011;91:328-32.

5. Kurek A, Johanne Peters EM, Sabat R, et al. Depression is a frequent co-morbidity in patients with acne inversa. J Dtsch Dermatol Ges 2013;11:743-50.

6. Jemec GB. Clinical practice. Hidradenitis suppurativa. N Engl J Med 2012;366:158-64.

7. Shah N. Hidradenitis suppurativa: a treatment challenge. Am Fam Physician 
2005;72:1547-52.

8. Kimball AB, Kerdel F, Adams D, et al. Adalimumab for the treatment of moderate to severe Hidradenitis suppurativa: a parallel randomized trial. Ann Intern Med 2012;157:846-55.

9. Amano M, Grant A, Kerdel FA. A prospective open-label clinical trial of adalimumab for the treatment of hidradenitis suppurativa. Int J Dermatol 2010;49:950-5.

10. Mekkes J. Weekly adalimumab significantly reduces the number of abscesses and inflammatory nodules in patients with moderate to severe hidradenitis suppurativa. Evid Based Med 2017; 22:34.

11. Ryan C, Sobell JM, Leonardi CL, et al. Safety of Adalimumab dosed every week and every other week: focus on patients with hidradenitis suppurativa or psoriasis. Am J Clin Dermatol 2018;19:437-47.

12. Seyed Jafari SM, Knusel E, Cazzaniga $\mathrm{S}$, Hunger RE. A retrospective cohort study on patients with hidradenitis suppurativa. Dermatology 2018:1-8.

13. Sbidian E, Hotz C, Seneschal J, et al. Antitumour necrosis factor-alpha therapy for hidradenitis suppurativa: results from a national cohort study between 2000 and 2013. Br J Dermatol 2016; 174:667-70.

14. Sartorius K, Emtestam L, Jemec GB, Lapins J. Objective scoring of hidradenitis suppurativa reflecting the role of tobacco smoking and obesity. $\mathrm{Br}$ J Dermatol 2009;161:831-9.

15. Jemec GB, Heidenheim M, Nielsen NH. The prevalence of hidradenitis suppurativa and its potential precursor lesions. J Am Acad Dermatol 1996;35:191-4.

16. van der Zee $\mathrm{HH}$, de Ruiter L, van den Broecke DG, et al. Elevated levels of tumour necrosis factor (TNF)-alpha, interleukin (IL)-1beta and IL-10 in hidradenitis suppurativa skin: a rationale for targeting TNF-alpha and IL1beta. Br J Dermatol 2011;164:1292-8.

17. Gulliver W, Zouboulis CC, Prens E, et al. Evidence-based approach to the treatment of hidradenitis suppurativa/acne inversa, based on the European guidelines for hidradenitis suppurativa. Rev Endocr Metab Disord 2016;17:343-51.

18. Martin-Ezquerra G, Masferrer E, Masferrer-Niubo M, et al. Use of biological treatments in patients with hidradenitis suppurativa. J Eur Acad Dermatol Venereol 2015;29:56-60.

19. Zouboulis CC, Desai N, Emtestam L, et al. European S1 guideline for the treatment of hidradenitis suppurativa/acne inversa. J Eur Acad Dermatol Venereol 2015;29:619-44.

20. van Rappard DC, Limpens J, Mekkes JR. The off-label treatment of severe hidradenitis suppurativa with TNFalpha inhibitors: a systematic review. J Dermatolog Treat 2013;24:392-404.

21. Kimball AB, Okun MM, Williams DA, et al. Two Phase 3 Trials of Adalimumab for Hidradenitis Suppurativa. N Engl J Med 2016; 375:422-34.

22. Sotiriou E, Goussi C, Lallas A, et al. A prospective open-label clinical trial of efficacy of the every week administration of adalimumab in the treatment of hidradenitis suppurativa. J Drugs Dermatol 2012;11:s15-20.

23. Arenbergerova M, Gkalpakiotis S, Arenberger P. Effective long-term control of refractory hidradenitis suppurativa with adalimumab after failure of conventional therapy. Int $\mathrm{J}$ Dermatol 2010;49:1445-9.

24. Blanco R, Martinez-Taboada VM, Villa I, et al. Long-term successful adalimumab therapy in severe hidradenitis suppurativa. Arch Dermatol 2009;145:580-4.

25. Yamauchi PS, Mau N. Hidradenitis suppurativa managed with adalimumab. J Drugs Dermatol 2009;8:181-3.

26. Miller I, Lynggaard CD, Lophaven S, et al. A double-blind placebo-controlled randomized trial of adalimumab in the treatment of hidradenitis suppurativa. Br J Dermatol 2011;165:391-8.

27. Ingram JR, Woo PN, Chua SL, et al. Interventions for hidradenitis suppurativa: a Cochrane systematic review incorporating GRADE assessment of evidence quality. Br J Dermatol 2016; 174:970-8.

28. Shavit E, Dreiher J, Freud T, et al. Psychiatric comorbidities in 3207 patients with hidradenitis suppurativa. J Eur Acad Dermatol Venereol 2015;29:371-6.

29. Scheinfeld N, Sundaram M, Teixeira H, et al. Reduction in pain scores and improvement in depressive symptoms in patients with hidradenitis suppurativa treated with adalimumab in a phase 2, randomized, placebo-controlled trial. Dermatol Online J 2016;22.

30. Kimball AB, Jemec GB, Yang M, et al. Assessing the validity, responsiveness and meaningfulness of the Hidradenitis Suppurativa Clinical Response (HiSCR) as the clinical endpoint for hidradenitis suppurativa treatment. Br J Dermatol 2014;171:1434-42.

31. Kimball AB, Sobell JM, Zouboulis CC, et al. HiSCR (Hidradenitis Suppurativa Clinical Response): a novel clinical endpoint to evaluate therapeutic outcomes in patients with hidradenitis suppurativa from the placebo-controlled portion of a phase 2 adalimumab study. J Eur Acad Dermatol Venereol 2016;30: 989-94. 